\title{
RELACIÓN ENTRE MARKETING Y UNIVERSIDAD. REVISIÓN TEÓRICA Y PROPUESTA DE UN MODELO TEÓRICO Y DE MARKETING 3.0
}

\section{RELATIONSHIP BETWEEN MARKETING AND UNIVERSITY. THEORETICAL REVIEW AND PROPOSAL OF A THEORETICAL AND MARKETING MODEL 3.0}

Doña Toledo, Luis (Universidad de Granada) *

Luque Martínez, Teodoro (Universidad de Granada) ${ }^{* *}$

\section{RESUMEN}

Las universidades aplican medidas de marketing en su gestión. La adopción de prácticas de marketing ha sido una respuesta a los múltiples cambios políticos, tecnológicos y sociales (así como un aumento de la competencia y la internacionalización) que afecta al mundo de la educación superior. En el presente trabajo se revisa el estado de la cuestión acerca de la gestión del marketing en la universidad. El objetivo es comprender la aparición y evolución de estas prácticas en la universidad y establecer un modelo conceptual que comprende cinco factores: personas, procesos, capacidades, calidad (premium) y proyección (imagen). Además, se propone un marketing de 3.0 que sirva para orientar la dirección del marketing dentro del sector de la educación superior.

Palabras claves: Marketing, política universitaria, stakeholders, gestión en la educación superior. JEL: A22, I2, I23, M3, M31, M38.

\begin{abstract}
The universities apply marketing measures in their management. The adoption of marketing practices has been a response to the many political, technological and social changes (as well as increased competition and globalization) affecting the world of higher education. In this paper the state of the question about marketing management at the university is reviewed. The goal is to understand the emergence and evolution of these practices in college and establish a conceptual model. People, processes, capabilities, quality (premium) and projection (image): five variables within the university marketing are proposed. In addition a model of Marketing 3.0 to serve to guide the direction of marketing within the higher education sector is proposed.
\end{abstract}

Key words: Marketing, university policy, stakeholders, management in higher education. JEL: A22, I2, I23, M3, M31, M38.

* Departamento de Comercialización e Investigación de Mercados, Facultad de Ciencias Económicas y Empresariales, Campus Universitario de Cartuja, 18071. luisdt@ugr.es

** Departamento de Comercialización e Investigación de Mercados, Facultad de Ciencias Económicas y Empresariales, Campus Universitario de Cartuja, 18071. tluque@ugr.es

Recibido: Julio de 2016. Aceptado: Septiembre de 2017. 


\section{INTRODUCCIÓN}

En los últimos años, el sector de la educación superior (ES) ha sufrido una importante transformación. A modo de síntesis, en Europa en las últimas décadas, se ha producido una mayor tasa de participación de la universidad en la economía, mayor internacionalización, creciente importancia de las economías dirigidas por el conocimiento y el aumento de la competencia global. Estos cambios han dado lugar a dos grandes políticas en Europa: el Proceso de Bolonia y la Estrategia 2020, así como el Programa de Modernización de la Educación Superior (Sursock, Smidt y Davies, 2010).

Todo ello sumado a una serie de circunstancias como son las dificultades a la hora de obtener financiación (Parameswaran y Glowacka, 1995; Helgesen y Nesset, 2007), la aparición de nuevas alternativas educativas propiciadas por el desarrollo de las tecnologías (como es el caso de los MOOC) o los cambios demográficos a nivel mundial han incentivado la búsqueda de nuevas fórmulas de gestión dentro del sector universitario.

Ello ha dado lugar a una nueva situación de incertidumbre (Maringe, 2004) que lleva a una mayor preocupación por adoptar una orientación al marketing y desarrollar relaciones duraderas con los graduados y las empresas (Mora, 1997). Durante los últimos años, el sistema financiero para instituciones de educación superior se ha ajustado en muchos países (OCDE, 2013, 2016). La financiación gubernamental es cada vez más escasa por lo que las universidades buscan vías alternativas de financiación desarrollando estrategias de negocio en las instituciones educativas (DeShields, Kara y Kaynak, 2005; Arena, Arnaboldi y Azzone, 2010).

El objetivo de este trabajo es contribuir al debate y reflexionar sobre la aplicación del marketing en la universidad y la educación superior. En definitiva, examinar la relevancia de la situación y evolución de las políticas y prácticas de marketing en el sector de la educación superior, formulando un modelo teórico que describa el papel del marketing en la educación superior.

Para este propósito, la metodología seguida consiste en un enfoque exploratorio, con la revisión de 104 publicaciones acerca de las políticas de marketing en la educación superior en el período 1970-2016. Los motores de búsqueda utilizados fueron las bases de datos científicas de EBSCOM, Proquest y Google Scholar. Como criterios de búsqueda se incluyeron obligatoriamente las palabras clave "higher education" o "university", junto con términos como "marketing”, "market orientation”, "education quality" "marketing planning” "consumer behaviour” y "strategic marketing”. Tras esta revisión sistemática se sintetizan y esquematizan los resultados de la investigación realizada mediante la propuesta del modelo conceptual y síntesis de los resultados de la literatura académica de forma cronológica.

\section{LA GESTIÓN EN EL SECTOR DE LA EDUCACIÓN SUPERIOR}

La educación de las próximas generaciones debe ser de mayor calidad, por ende, debemos esforzarnos en optimizar los recursos y hacer una gestión universitaria de mayor calidad y eficiencia (Díaz-Canel, 2011). La UNESCO (1998) afirma que "las instituciones universitarias están obligadas, más que nunca, a implementar modernos enfoques de dirección en su práctica diaria y a utilizar los recursos de que disponen con gran eficacia y eficiencia. Estas circunstancias, así como las que se derivan de la necesidad de una mayor coordinación y racionalidad para dar respuesta a los nuevos requerimientos que imponen las exigencias multidisciplinarias de los procesos y de una mayor competencia por el uso de recursos comunes, le imprimen un sello característico a la gestión universitaria de estos tiempos”. 
Dada la naturaleza cambiante del sector de la educación superior, es aconsejable que los gerentes y líderes de las universidades adopten un enfoque centrado en el usuario. Cada vez más, las instituciones de educación superior se reconocen como una industria de servicios y, por lo tanto, están poniendo mayor énfasis en la satisfacción del cliente y una adecuada gestión de la universidad (Deshields, Kara y Kaynak, 2005). Tradicionalmente, las instituciones de educación superior han carecido de una consolidada cultura de gestión, sin embargo, la mayoría de ellos aplican estrategias de gestión y comercialización diariamente, a menudo inconscientemente, y sin un conocimiento preciso del proceso que realizan (Kirp, 2003; Ross, Grace y Shao, 2013).

Arnoldo y Nicolás (2001) consideran que una adecuada gestión de la universidad debe comprender las siguientes estrategias con el objetivo de crear una ventaja competitiva sostenible en el entorno actual de las instituciones de educación superior y en la cual reconocen la importancia del papel del marketing: (1) estrategia financiera para una asignación y aprovechamiento de los recursos; (2) estrategia de recursos humanos, que incluye tanto a los docentes como al personal administrativo; (3) estrategia tecnológica. En este concepto se incluye, por ejemplo, el equipamiento audiovisual de las salas de clases, bibliotecas y equipamiento de laboratorios; (4) estrategia de adquisiciones, que se aplica a todo lo relacionado con el área de adquisiciones para la operación interna; (5) estrategia de servicio, que define los objetivos estratégicos del producto o servicio que la unidad entrega en términos de costo, calidad, confiabilidad, flexibilidad e innovación; (6) estrategia de marketing, que promueve las políticas de la institución respecto a las acciones para captar alumnos de pregrado y postgrado tanto nacionales como internacionales y anunciarse de manera propicia al medio externo. Según Santesmases (1996) una estrategia de marketing supone un modo de "concebir y ejecutar la relación de intercambio con la finalidad de que sea satisfactoria a las partes que intervienen y a la sociedad, mediante el desarrollo, valoración, distribución y promoción, por una de las partes de los bienes, servicios o ideas que la otra parte necesita”. El marketing, desde una perspectiva clásica, supone llevar a cabo las estrategias de precio, producto, distribución y comunicación. Más tarde se discutirá acerca de cómo los investigadores conciben si esas u otras estrategias deben ser las herramientas dentro del sector universitario.

La estrategia de marketing se integraría dentro de una planificación estratégica de la universidad. La planificación estratégica consiste en la formulación, ejecución y evaluación de acciones que permiten que una organización logre sus objetivos (David, 2003). Munuera y Rodríguez Escudero (2007) siguiendo las premisas de Narver y Slater (1990) indican que la gerencia estratégica busca concentrarse en sólo aquellos objetivos factibles de lograr y en qué negocio competir de acuerdo a las oportunidades y amenazas del entorno.

La aplicación de la planificación estratégica puede ser implementada tanto en organizaciones públicas como privadas y así beneficiarse de las ventajas que se derivan de tal planificación siempre teniendo en cuenta la naturaleza y las características del servicio y del sector donde compite la institución. En el sector de las IES (Instituciones de Educación Superior), la planificación resulta esencial para lograr los objetivos, al igual que en cualquier otra empresa u organización de servicios.

La planificación estratégica es un pilar fundamental dentro de la gestión universitaria pues permite una serie de beneficios (Luque y Del Barrio, 2005): evita los problemas asociados con la inmediatez y anticipa los cambios en el entorno, dinamiza los sistemas de gestión, ayuda a priorizar la gestión, fomenta un proceso de aprendizaje y desarrollo organizativo y facilita la comunicación entre la universidad y los distintos colectivos a los que se dirige. Tal como afirma Steiner (1979) no se trata de una planificación estática y que 
sustituya el juicio e intención de los gestores, sino que es un ejercicio de reflexión donde establecer metas y los métodos para alcanzarlas.

Por su importancia, las planificaciones estratégicas han ido desarrollándose de forma notable en los países anglosajones (Universidad de California, Glasgow, Leeds, Sydney...) así como en España (Universidad del País Vasco, Granada, Jaén, Autónoma de Madrid, Barcelona, Pública de Navarra, etc.).

La planificación estratégica comprende la estrategia de marketing de la universidad, formulada a partir de un diagnóstico, con especial interés en los diferentes públicos implicados.

\section{3. ÓPTICA DE MARKETING DE LAS INSTITUCIONES UNIVERSITARIAS}

El fenómeno de la globalización ha impulsado la mercantilización de la educación superior (Steger, 2003). El impacto de la globalización en la universidad se caracteriza por el incremento de los índices de matriculación, la menor financiación pública y la aparición del paradigma del aprendizaje a lo largo de la vida (Dale, 2007). Este fenómeno y la búsqueda continua de la mejora de la gestión y el servicio universitario, ha provocado que en los últimos años la universidad haya adquirido nuevas tácticas desde una perspectiva del marketing que le permita actuar de forma más eficaz y planificada, así como adaptarse al nuevo entorno competitivo.

También la crisis de lo público y la erosión de la confianza de la sociedad en la educación se han manifestado en constantes retos planteados a la eficiencia, la productividad, la equidad y la calidad de los grandes sistemas educativos europeos (Díaz Barriga, 1998). La reciente crisis económica y financiera de las universidades ha ido acompañada, como causa y consecuencia a la vez, por una redefinición constante y continua del sentido de la universidad, los objetivos de la misma y de las prácticas necesarias de la educación superior (Ordorika, 2008). La ola de liberalización generalizada también ha afectado a las instituciones de educación superior y, por ello, los gobiernos de todo el mundo empezaron a reducir el gasto de las instituciones. De este modo, las universidades tuvieron que asumir gradualmente la responsabilidad financiera de la gestión de sus propias organizaciones llevando a plantear el tema de la orientación al marketing en las universidades (Maringe y Gibbs, 2009). A pesar de esta presión financiera, en muchos países y universidades (i.e. la mayoría de los países europeos, como España) la financiación proviene todavía en su mayor parte de la administración pública.

Un enfoque orientado al marketing eficiente genera una serie de beneficios para las IES y sus públicos, tanto económicos como no económicos, en términos de desarrollo social, ético y equilibrio social (Luque y Del Barrio, 2009). Hay una serie de estudios que confirman que la incorporación de la orientación al marketing de las universidades contribuye a mejorar su competitividad y sus resultados posteriores (Litten, 1980; Narver y Slater, 1990; Qureshi, 1993; Taylor y Reed, 1995; Caruana, Ramaeshan y Ewing, 1998; Umashankar, 2001; Czarniawska y Genell, 2002; Maringe y Foskett, 2002; Bok, 2003; Jongbloed, 2003; Arnett, German y Hunt; 2003; Ivy y Naude, 2004; Hemsley-Brown y Oplatka, 2006; Voon, 2006; Hayes, 2007; Newman y Jahdi, 2009; Akonkwa, 2009; Maringe y Gibbs, 2009; Hemsley-Brown y Oplatka, 2010; Maringe y Mourad, 2012; Ross et al., 2013).

La AMA (Asociación Americana de Marketing) define en el año 2013 el concepto de marketing como "la actividad, conjunto de instituciones y procesos para crear, comunicar, distribuir e intercambiar ofertas que tengan valor para los consumidores, los clientes, los socios y la sociedad en su conjunto”. Dicha definición se aleja de las creencias del marketing 
como una mera herramienta comercial pues se remarca la importancia del servicio a la sociedad y no sólo de los clientes, algo que es demandado continuamente a la ES desde diferentes esferas de la sociedad y justificaría su pertinencia como óptica dentro de las universidades, pues el marketing debe satisfacer de forma coordinada los intereses de todos los públicos o consumidores y moldear los mejores productos y servicios según su requerimientos (Kotler, 2000). Además, la propia definición resalta la creación de valor, pues la universidad debe aportar un valor añadido a los distintos públicos y al desarrollo humano y social.

Kotler y Fox (1985, p. 6) en su libro "Marketing Estratégico para Instituciones Educativas" definen al marketing en el contexto de la educación como "el análisis, la planificación, la implementación y el control de programas cuidadosamente formulados y diseñados para llevar a cabo intercambios voluntarios de los valores con un mercado objetivo para lograr así los objetivos organizacionales. El marketing consiste en el diseño de una estrategia institucional que satisfaga las necesidades y deseos del mercado objetivo y el uso eficaz del precio, comunicación y distribución para informar, motivar y dar servicio a esos mercados”. Tales autores señalan que el marketing de las universidades debe tener una función esencial: la función social pues la universidad debe preservar los intereses de la sociedad a largo plazo.

La idea de las universidades como amplios proyectos culturales de la sociedad o como instituciones dirigidas a la producción de bienes públicos ha pasado de ser un discurso meramente marginal o discursivo (Readings, 1996; Marginson, 1997). Esta idea ha sido sustituida por un renovado énfasis entre los vínculos de la universidad y las políticas de mercado (Margison y Considine, 2000), por un esquema de universidades emprendedoras (Clark, 1998), por nociones de excelencia (Readings, 1996), por conceptos y objetivos de dirección como "productividad" o "eficiencia”, o por la creciente privatización de la financiación universitaria y el crecimiento de la competitividad (Slaughter y Leslie, 1997).

Entre los principales motivos por los cuales la universidad comenzó a aplicar, de forma más explícita, conceptos propios de marketing se pueden destacar los siguientes:

- Nueva gestión pública: las universidades aplican cada vez más políticas supranacionales (por ejemplo, en Europa) y existe una creciente competencia entre las universidades (Akonkwa, 2009).

- Masificación: la población universitaria mundial se duplicó entre 1975 y el año 2000.

- Profesionalización de la enseñanza superior: preparar adecuadamente a los estudiantes para que estén listos para el mercado de trabajo.

- Reducción de la financiación pública.

- Toma de conciencia de problemas de gestión de la calidad (Akonkwa, 2009).

- Auge de la “servucción”. El avance en la gestión de los servicios.

Un estudio realizado mediante la técnica de paneles por Hayes (2007) a 20 expertos en educación universitaria revelaba la futura evolución de la denominada "comercialización de la educación superior”. Los panelistas coincidían en que las prácticas de marketing serían el pilar estratégico debido a cinco razones fundamentalmente:

1. La competencia por los estudiantes se incrementará debido a una crisis demográfica del número de estudiantes en edad universitaria. 
2. Los estudiantes y los padres pondrán más énfasis en el valor ofrecido, debido a los incrementos de precios.

3. Las universidades han de alcanzar sectores no tradicionales donde no se percibe tan claramente el beneficio ofrecido por la universidad.

4. Un aumento constante de alternativas de prácticas de mercado se difunde rápidamente en las instituciones sin ánimo de lucro.

5. Las instituciones privadas podrán experimentar una mayor competencia por parte de las instituciones públicas que están adoptando rápidamente sus propias técnicas de marketing y actúan progresivamente como "semi-privadas".

Todo esto ha dado lugar a que cada vez se dan con mayor frecuencia en la educación superior palabras o términos como satisfacción de estudiantes, retención, intención, cuota de mercado o valor percibido (Homburg y Pflesser, 2000). Esta situación conlleva la petición de una mayor investigación que se centre en la literatura de marketing educativo y la aplicación de políticas de marketing en la educación superior (Hemsley-Brown y Oplatka, 2010).

\subsection{Orientación al marketing en la educación superior}

Recordando lo que se ha comentado anteriormente, diferentes factores han dado lugar a la aparición del "marketing universitario" o la inclusión del término "productos" en el sector de la educación superior. Así, la adopción de prácticas de mercado o enfoque estratégico del marketing se ha convertido en una de las características destacada de la educación superior contemporánea (Slaughter y Leslie, 1997; Ordorika, 2002, 2004).

Litten (1980) fue uno de los primeros autores que abordó el tema de la perspectiva del marketing y la orientación al mercado en el sector de la educación superior. En su estudio establecía las diferencias entre el marketing de organizaciones y empresas de negocio con respecto a las universidades. Entre esas diferencias se encontraban el hecho de que la selección de la universidad por parte del estudiante supone un largo y complejo proceso dado las importantes consecuencias de que esta elección se deriva. No se trata de una elección basada únicamente en la conveniencia o en el precio pues a diferencia de otros tipos de negocios, la organización (la universidad) no espera que el beneficio proceda fundamentalmente de la lealtad y de la repetición de compra, sino que espera que el alumno, con una sola elección, cumpla todas sus altas expectativas. Además, el estudiante asume todo el servicio de la organización con sus debilidades y fortalezas, por ejemplo, no elige una universidad que sea la mejor en la enseñanza de idiomas y otra para aquella que ofrezca un mejor servicio en prácticas (aspectos que sí suceden la mayoría de servicios o productos). Así mismo, el propio Litten (1980) advertía de que el futuro de las universidades se basaría en la reputación e imagen de las mismas debido a las pocas diferencias que existen en la formación teórica entre universidades, así como la gran sensibilidad dentro de la educación superior para términos como marketing o gestión del servicio. Estos obstáculos deberían ser superados por los gestores y los académicos para conseguir una verdadera orientación al mercado u orientación al marketing ${ }^{1}$.

\footnotetext{
${ }^{1}$ Es importante señalar que los conceptos de orientación al mercado y orientación de marketing han sido indistintamente usados por la mayoría de los autores, que parecen no encontrar diferencias importantes entre ambos conceptos (Shapiro, 1998). McCarthy y Perreault (2007) afirman que la orientación al mercado es la implantación de la filosofía de marketing en la empresa por lo que son conceptos similares y que la literatura ha empleado indiscriminadamente en la mayoría de los casos ambos términos de forma análoga.
} 
Este término de orientación al mercado fue concebido para reflejar la aplicación práctica del concepto de marketing (Kohli y Jaworski, 1990), expresa la necesidad sustancial de una organización para poder competir en el sector donde opera (Lafferty y Hult, 2001). La premisa de esta orientación de marketing es que el desempeño organizacional depende de la capacidad de identificar y satisfacer las necesidades del cliente (Kirca, Jayachandran y Beardon, 2005) así como de una relación positiva entre la orientación al mercado y el desempeño de la organización (Narver y Slater, 1990).

Se puede decir que una organización orientada al marketing es una organización orientada hacia el aprendizaje, lo que refuerza sus creencias y comportamientos a través de los principios del aprendizaje organizacional (Jaworski y Kohli, 1996). Se ha demostrado que las organizaciones con una fuerte orientación hacia el marketing o al cliente tienen una mayor capacidad para identificar los cambios en la demanda, anticipar oportunidades y responder a la competencia, obteniendo así mejores resultados (Narver y Slater, 1990; Cann y George, 2003).

En la literatura predominan dos concepciones de orientación de mercado (Homburg y Pflesser, 2000): la que adopta el punto de vista de procesamiento de la información (Kohli y Jaworski, 1990), y la que adopta un punto de vista cultural (Narver y Slater, 1990). La primera concibe la orientación al mercado como una evolución de la generación, la difusión y la respuesta a los cambios que se dan naturalmente en el mercado. La perspectiva cultural destaca la orientación al mercado como la capacidad de aprender acerca de los clientes y de los competidores con el fin de responder a las tendencias, así como conocer y responder a las influencias del entorno. Por lo tanto, la orientación al mercado es una característica organizacional implícita, en lugar de un comportamiento explícito pues muchas organizaciones llevan a cabo actividades propias del campo del marketing aunque de forma casi inconsciente (Ross et al., 2013).

Así pues, resulta lógico afirmar que la educación superior lleva a cabo una orientación al mercado de tipo cultural pues son muchas las políticas gubernamentales o los informes internacionales que exigen a las universidades adaptarse, satisfacer a sus distintos públicos y anticiparse a los cambios además de analizar el valor de la organización. Esta perspectiva exige cuestionarse a la institución porqué un "cliente" elige tal organización, producto o servicio. Esta perspectiva comprende tres dimensiones (Narver y Slater, 1990) que han sido analizadas por distintos autores dentro de la educación superior (Siu y Wilson, 1998; Flavián y Lozano, 2004; Hemsley-Brown y Oplatka, 2006; Hemsley-Brown y Oplatka, 2010; Ross et al., 2013):

- Orientación a la competencia: la necesidad de analizar el entorno, así como las fortalezas y debilidades de la organización. Ello se logra únicamente con el hecho de que los profesores y el personal de administración comprendan la importancia de esta filosofía organizacional ya que daría lugar a iniciativas y mejoras innovadoras que crearían servicios adicionales a los diferentes públicos de la universidad (Hemsley-Brown y Oplatka, 2010).

- Orientación al "cliente": implica comprender de dónde proceden sus fuentes de satisfacción cumpliendo sus expectativas. Lo más característico es que no se trata de una conducta concreta frente a un cliente real, sino de una actitud permanente de contar con las necesidades y demandas del cliente. Supone recoger información continuamente acerca de las características de los clientes e implica que la experiencia de los mismos esté en el centro de las prioridades de la organización. Tal como afirman Hemsley-Brown y Oplatka (2010) en la educación superior 
requiere adaptar la enseñanza a los estudiantes y estar atentos a sus diferentes puntos de vista además de tener en cuenta sus diferentes públicos.

Sin embargo, a lo largo de los diversos estudios, los principales investigadores han puesto en duda el hecho de considerar como cliente a quienes se benefician del servicio universitario (empresas, estudiantes, sociedad, etc.) pues se trata de un servicio público, además de las implicaciones académicas, sociales, intelectuales y culturales que conllevan las instituciones universitarias. Existe un debate sobre quiénes son los grupos susceptibles de considerarse públicos de la universidad (Redding, 2005; García Sanchís, 2012) siendo el concepto de públicos o de agentes, en lugar de clientes, términos más apropiados para el sector universitario.

- Coordinación inter-funcional: se refiere a la creación de un valor superior para sus públicos. Es la fuente principal de la ventaja competitiva. La combinación de los diferentes servicios exige una misma cultura organizacional teniendo en cuenta tanto a los públicos externos como internos de la universidad (Siu y Wilson, 1998).

Centrándonos en estudios que hayan analizado la relación entre marketing y universidad de manera más pormenorizada, en primer lugar, podemos señalar a Wasmer y Bruner (2000) que analizaron las características principales que tienen las universidades con una perspectiva de marketing. Las universidades pequeñas, con menor número de estudiantes tienen una cultura y misión organizacional más proclive al marketing dado su necesidad de atraer nuevos estudiantes. Las grandes universidades se centran en aspectos más académicos. Además, eran las universidades privadas quienes tenían una verdadera cultura organizacional de mercado debido a su propia naturaleza además de que existía correlación entre las políticas de marketing y aquellas universidades caracterizadas por la innovación.

Otros estudios (Hemsley-Brown y Oplatka, 2006; Hemsley-Brown y Oplatka, 2010) analizaron las diferencias que existen internacionalmente en la aplicación de prácticas de marketing según los países. Las naciones cuyas universidades son más recientes (inauguradas fundamentalmente en los últimos veinticinco años del siglo XX) son las que llevan a cabo más actividades orientadas al marketing.

En la configuración del marketing dentro de la universidad, Ivy y Naude (2004) analizaron los componentes o marketing mix que deben integrarse en la universidad. Aunque su estudio estaba dirigido únicamente a másteres de negocios establecieron las 7 P's del marketing aplicables a la universidad: "premium” (alta calidad, entrega de servicios de excelencia); "proyección” (imagen adecuada y percepción positiva y diferenciada entre los públicos); "publicidad”; "precio"; "programa de estudios”; "prospectus” (fuentes de información); y “personas” (éste último coincide con los estudios de Booms y Bitner (1981) y Newman y Jahdi (2009) que se comentan en el siguiente párrafo).

Newman y Jahdi (2009), teniendo en cuenta la creciente importancia y la aplicación de políticas de marketing, analizaron el impacto de estas prácticas entre los profesores y los estudiantes. Los autores sugieren que la educación superior no es un sector susceptible de aplicar las cuatro P's clásicas del marketing mix (producto, precio, comunicación y distribución). Siguiendo las teorías de Booms y Bitner (1981) acerca de las tres P's del marketing de servicios (personas, procesos y evidencia física, ésta última referida a la imagen corporativa) sugieren sustituir las "personas” por “valías”, los procesos por “capacidad” y la evidencia física por "carisma”. En este caso las personas supondrían el análisis constante de los estudiantes, ya que la universidad es un servicio que exige una gran confianza e implicación mutua entre el estudiante con el profesor y el personal de administración y servicios. Las “capacidades” (el proceso) se refiere a la facilidad de obtención de información, 
la comunicación entre la institución y el estudiante potencial, así como la inscripción y la organización de conferencias. Las “capacidades” de una institución pueden convertirse en una gran ventaja competitiva. Por último, el "carisma” (la evidencia física) es la representación visual de una entidad, así como su ubicación, instalaciones y servicios.

Ross et al. (2013) analizaron la importancia de la orientación al marketing para el reclutamiento y atracción de estudiantes internacionales, como respuesta a la creciente globalización de la educación superior. En su estudio concluían que la orientación al marketing de las universidades requería de la compresión de las necesidades, las motivaciones y las expectativas de los estudiantes potenciales y futuros para dar lugar realmente a una verdadera satisfacción.

\subsection{Debate acerca de la estrategia de marketing en las IES}

La política de marketing en el marco de la Educación Superior es un área en desarrollo, tanto a nivel conceptual como operativo. La comercialización de programas educativos y el desarrollo de estrategias de marketing relacional ha atraído la atención de los investigadores en los últimos años que han tratado de averiguar cuáles con las consecuencias de las prácticas de marketing en el sector universitario (Hayes, 1996, Gibbs, 2001).

Sin embargo, el marketing no siempre es bien visto dentro de las IES (Oplatka, 2009) ya que el concepto ha sido malentendido durante largo tiempo en la educación (Maringe, 2004). En la literatura, hay un desacuerdo en cuanto al papel del marketing en las IES con algunos argumentando que desempeña un papel crucial en su rendimiento (Oplatka y HemsleyBrown, 2007), mientras que otros cuestionan su idoneidad (Khalifa, 2010; Ramachandran, 2010). Por ejemplo, Svensson y Wood (2007) afirmaban que la universidad no puede adoptar una perspectiva de marketing pues supone la pérdida de control por parte de los académicos y profesores de las decisiones. No es la universidad la que debe adaptarse continuamente a los estudiantes debido a que muchos de ellos no tienen un compromiso o implicación suficiente y es la universidad la que debe decidir la formación adecuada para cada titulación. Smith (1997) argumenta que la mercantilización de la educación superior no es más que "un ataque concertado contra las estructuras y valores tradicionales de estas instituciones". McGrath (2003) afirma que la universidad no debe evaluarse según su número de estudiantes, capacidad de reclutamiento, sus estrategias de marketing o imagen, sino que la calidad universitaria debe evaluarse únicamente sobre la base de los resultados alcanzados laboralmente por parte de los estudiantes. Sin embargo, la afirmación del último autor tiene ciertas limitaciones puesto que no considera las características del entorno no sujetas al control de las universidades como son las oportunidades laborales o crisis de empleo. Es decir, aún con las mismas políticas, los mismos docentes y las mismas instalaciones pueden diferir ampliamente los resultados logrados por los estudiantes, muchas veces ajenos e incontrolables por la universidad según épocas de prosperidad o de crisis económica. De este modo, los logros laborales no son un indicador de la calidad de la experiencia en ciertos momentos. Además, un enfoque basado en los resultados obvia todos los aspectos y roles circundantes a la universidad: su papel abarca desde la promoción de valores y desarrollo humano y social hasta la contribución al tejido productivo que también son valorados en términos de calidad.

El mayor problema a la hora de implementar una estrategia de marketing en el ámbito de la gestión universitaria reside en la escasa o limitada comprensión por parte de los responsables de tal gestión sobre estrategias de mercado (Newman, 2002; Maringe, 2005), principalmente en las universidades públicas con mayor reticencia a aplicar principios del 
marketing, aunque ello es necesario para atender a la rendición de cuentas a la sociedad (Michavila, 2001). Otro importante obstáculo es que en muchas instituciones la filosofía del marketing se encuentra "bajo sospecha" al ser un concepto procedente del mundo de los negocios (Naude y Ivy, 1999; Maringe, 2005) y no es apropiada dada su naturaleza comercial (Wonders y Gyure, 1991). Incluso se le asocia con una simple herramienta de captación de estudiantes y generar ingresos (Conway, Mackay y Yorke, 1994). Newman y Jahdi (2009) afirman que la dificultad reside en que existe una gran diferencia entre la filosofía o retórica de marketing en la universidad con respecto a su real aplicación.

Hayes (2007) señalaba que el principal desafío del marketing en el sector de la educación superior es la resistencia a su implantación y práctica. Dicha conclusión se realizó a través de una comparación entre las clínicas médicas y profesionales de la salud con el propio sector universitario. En el inicio del marketing en el sector sanitario se temía que éste perjudicase la propia naturaleza e integridad de la medicina. Con el tiempo, el marketing se convirtió en una herramienta invaluable para asegurar la supervivencia y el éxito final de muchas instituciones de salud. Cada año, la aceptación creció y se amplió en tal campo. En consecuencia, muchos gestores universitarios confiaban en que se produciría el mismo patrón en la educación superior (Blackburn, 1980; Topor, 1983; Kotler y Fox, 1985).

Hutton (2002) señala que muchas instituciones públicas han implementado erróneamente sus estrategias de marketing pues lo han concebido únicamente como una herramienta empresarial obviando la idiosincrasia de cada sector y sus diferentes públicos. Tal como nos indica Gibbs y Knapp (2002) el marketing debe estar basado en las personas a las que se dirige, especialmente en campos como la educación o la salud.

Las instituciones de educación superior (ya sean públicas o privadas) no pueden ser tratadas como organizaciones puramente comerciales (Akonkwa, 2009). Sus misiones, su cultura y su estructura requieren un tratamiento especial para la implementación de la estrategia de marketing (Sirvanci, 1996, 2004). Según lo recomendado por la American Marketing Association (AMA) la estrategia de marketing se desarrolla de acuerdo al contexto donde prevalece el sector. En este sentido se debe tener en cuenta la idiosincrasia de la educación superior.

Otro problema es el obstáculo que supone cambiar la cultura organizativa de una manera que integre a todas las personas que forman parte de la organización (profesores y personal no docente), y tener que aceptar e incorporar la orientación al marketing en su marco mental y su estilo de trabajo. Actualmente, existe la necesidad de que las autoridades educativas y los equipos gestores de los centros dirijan sus actuaciones en dos direcciones fundamentales: orientarse al marketing y anticiparse a los factores ambientales (económicos, políticos, legales) que fomenten esta perspectiva con el objetivo de mejorar el funcionamiento de la universidad pública (Flavián y Lozano, 2004). El resultado de este sistema de gobierno es que los objetivos, las decisiones y medidas adoptadas por la universidad, por lo general, tratan de satisfacer los intereses de los clientes internos pero tienden a descuidar las necesidades de los estudiantes, las familias, las empresas y las instituciones gubernamentales.

Aquí es importante señalar que otro de los problemas ha sido la delimitación de los públicos (stakeholders) a los que se dirige la universidad. La educación superior tiene un número de considerable de públicos (empresas, estudiantes, padres, egresados, etc.), lo que puede dificultar la identificación de las necesidades y deseos de los públicos y el correcto desempeño institucional (Conway et al., 1994). Resolver esta cuestión es necesario para una adecuada formulación del plan de marketing, de calidad y de planificación estratégica de las universidades (Pereira y Silva, 2003). 
En resumen, hay reticencia en muchas instituciones universitarias a nociones como “clientes”, “mercado" o "ventaja competitiva”. Por el contrario, Pulido (2009) afirma que a las universidades les ha llegado el momento de vender mejor sus productos y servicios (docencia, investigación, conocimiento y servicio al tejido productivo) para convertirse progresivamente no sólo en productores sino también en comunicadores. A pesar de las dificultades, Hemsley-Brown y Oplatka (2006) afirman que la orientación al marketing de la educación superior se ha consolidado como un fenómeno global, en particular en países como Reino Unido, Estados Unidos, Canadá y Australia. Argumentan que resulta ilógico obviar la competencia creciente que existe entre las universidades, las dificultades de adaptación a las universidades o la importancia de satisfacer las distintas esferas de la sociedad. En palabras de los autores "la orientación al marketing no supone únicamente folletos publicitarios de papel satinado, sino que debe involucrar a cada una de las personas que trabajan en la institución”.

En este sentido, una óptica orientada al marketing se presenta como un recurso fundamental para crear una ventaja sostenible en el tiempo y mantener y mejorar la producción (Hult y Ketchen, 2001), algo que se ha intentado aplicar desde el mundo académico, sobretodo, desde principios de los años ochenta especialmente en universidades americanas (Bardo, Ross y Headley, 1990; Kotler y Fox, 1995). Así, ha habido un aumento progresivo de la preocupación por adaptarse al mercado en los últimos años (Capelleras y Veciana, 2001; Ressler y Abratt, 2009; Perin et al., 2012). Incluso ciertos estudios han mostrado el efecto positivo del marketing en la ES (Caruana et al., 1998) o su utilidad para la creación de valor de la universidad en su servicio a la sociedad (Bok, 2003). Muchos autores apoyan la idea generalizada de que ahora las instituciones de educación superior pueden ser consideradas organizaciones de servicios (Dolinsky, 1994; Kotler y Fox, 1995; Zammuto, Keaveney y O'Connor, 1996; Joseph y Joseph, 1997), por lo que sería pertinente la adopción de estrategias propias del sector servicios, entre ellas, el marketing.

Todo ello nos hace pensar que tal como afirma Kotler (2000) que en el caso de las universidades y otras entidades públicas la orientación no deber ser tanto al mercado sino una “orientación a la sociedad” dado los diversos públicos a los que se dirige. Ello estaría en línea con el enfoque de responsabilidad social que constituye una extensión del marketing clásico (Kotler, 1972) y que intenta equilibrar los beneficios empresariales, la satisfacción de los consumidores y la rendición de cuentas a la sociedad. Bajo esta óptica, Hayes (2007) estableció el triple beneficio que obtienen las universidades al adoptar una filosofía propia del marketing: (1) ayuda a la planificación organizacional; (2) el marketing utiliza la información para la toma de decisiones. La recogida constante de información y la evaluación de datos primarios y secundarios permiten tomar conciencia de la imagen y los resultados de la universidad. El marketing permite actuar de una manera más eficaz y eficiente; (3) el resultado de una adecuada combinación de marketing es la comunicación de la misión de la institución, la visión y los valores, tanto a los públicos internos como externos.

Teniendo en cuenta estas reflexiones, en la figura 1 se representa un modelo de marketing en la educación superior, que incluye los principales aspectos para su aplicación.

De forma detallada, el modelo propuesto integra los factores de contexto, tanto externos (internacionalización, mayor competitividad, búsqueda de financiación, etc.), como internos (búsqueda de un mejor desempeño enfocado a la calidad, imagen o satisfacción) que afectan al desempeño de las universidades. 


\section{FIGURA 1: MODELO CONCEPTUAL DE MARKETING UNIVERSITARIO}

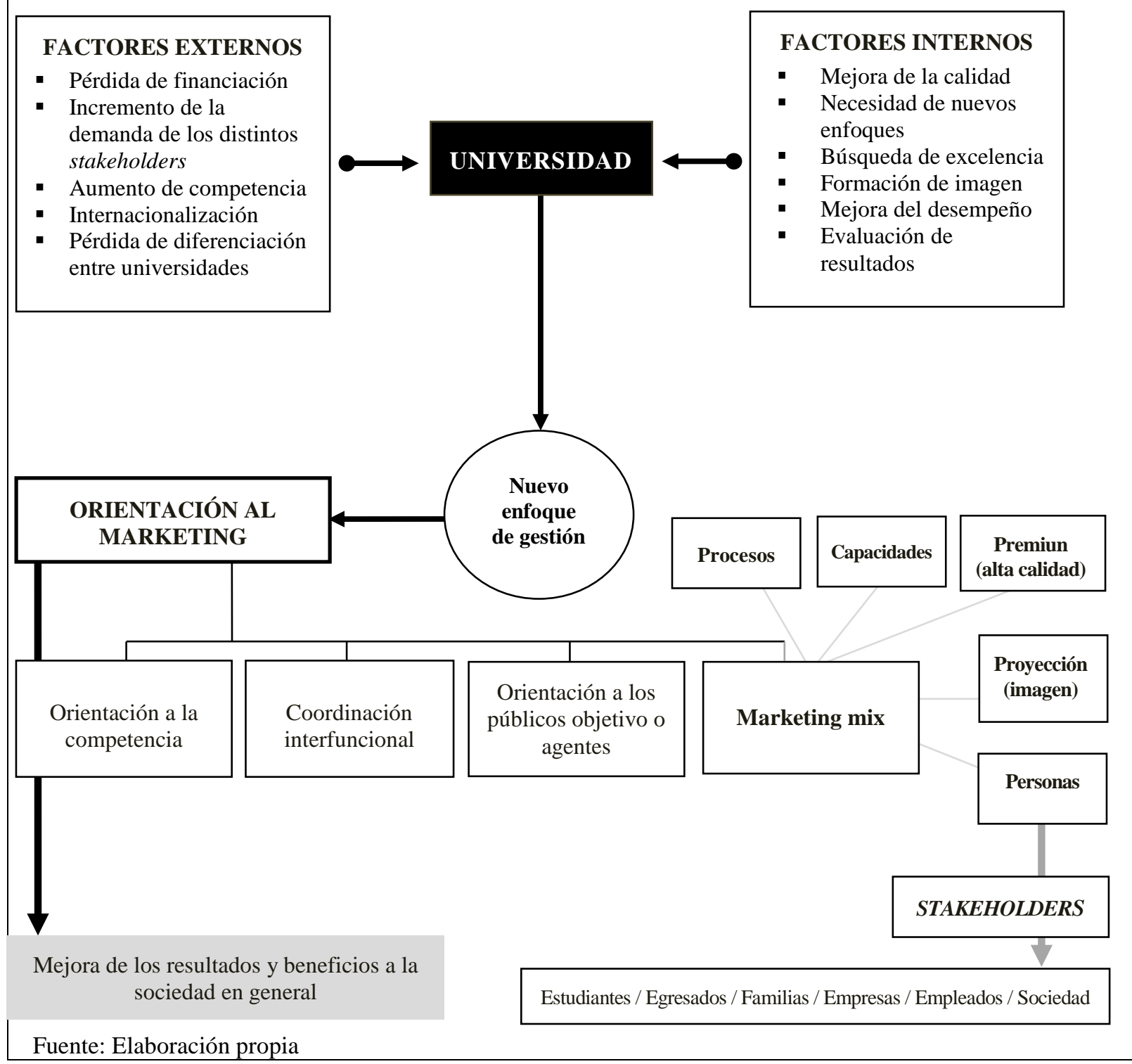

Así mismo, tales factores han dado lugar a un nuevo enfoque de gestión orientado al marketing que debe integrar las dimensiones de orientación a la competencia (análisis del entorno), coordinación interfuncional (generar valor) y orientación al cliente (genera satisfacción y que se sustituye por orientación a los distintos públicos, stakeholders), desarrollados y propuestos por diferentes autores (por ejemplo, Siu y Wilson, 1998; HemsleyBrown y Oplatka, 2010; Ross et al., 2013). Además, se propone desde este trabajo la integración de la dimensión de marketing mix. Siguiendo las directrices de autores como Booms y Bitner (1981), Ivy y Naude (2004) y Newman y Jahdi (2009) las 4 P’s clásicas del marketing no son aplicables a la idiosincrasia del ámbito universitario. De este modo, nuestro modelo propone la integración de los aspectos de:

1. Procesos: implica los componentes derivados de la institución teniendo en cuenta las diversas prestaciones que realiza. Comprende los diferentes aspectos que 
conforman la experiencia universitaria como la formación, asesoramiento académico, oferta de cursos, etc.

2. Personas: incluye todo tipo de interacción con el personal con el que el estudiante tiene contacto de una u otra forma. El profesorado (con el que el alumno tiene una mayor vinculación y deposita más expectativas), el personal de administración y servicios que incluye empleados de diferentes centros, departamentos o servicios auxiliares: secretaría, unidades de apoyo al estudiante, empleados de centros deportivos, etc. Además es importante tener en cuenta en este caso la problemática referida a los stakeholders a los que se dirige la universidad. Kotler y Fox (1985), Conway et al. (1994), Owlia y Aspinwall (1997) y Eagle y Brennan (2007) señalan entre otros stakeholders: estudiantes, padres, egresados, medios de comunicación, sociedad, proveedores, empresas, empleados, estudiantes potenciales y el gobierno.

3. Capacidades: capacidad de reclutamiento, organización y comunicación entre la institución y el estudiante potencial, así como su inscripción y ayuda a la inserción laboral, incluyendo así la capacidad de la universidad para que el alumno obtenga resultados de su formación.

4. Premium: excelencia en la entrega del servicio y gestión de la calidad del servicio universitario. Búsqueda de la diferenciación y la especialización.

5. Proyección (imagen): con el propósito de distinguirse de los competidores, las universidades han aumentado sus esfuerzos en desarrollar una imagen "de prestigio” (Beerli, Díaz y Pérez, 2002; McPherson y Schapiro, 1998). Maringe y Gibbs (2009) afirmaban que la imagen universitaria favorable tiene un impacto positivo en la percepción de un sistema educativo o, incluso, una sociedad. Como ejemplos mencionaban el compendio “Oxfbridge” de la imagen universitaria inglesa o el binomio Harvad-Yale como ejemplos de una imagen sólida basada principalmente en la investigación y la innovación tecnológica.

El modelo se ofrece como un resumen de toda la investigación académica y como aportación conceptual de la integración de los distintos factores del marketing universitario que concluye con el resultado de este tipo de gestión, como es la mejora de los resultados y la obtención de beneficios.

A continuación (Cuadro 1) se recogen los principales estudios o investigaciones que han abordado la temática de la orientación al marketing, así como sus ventajas e inconvenientes en la educación superior y su principal aportación sobre el asunto. Se puede observar que la mayoría de ellos se han desarrollado a partir del año 2000, lo que sugiere la creciente importancia del fenómeno a pesar del reconocimiento de la orientación al marketing desde hace varias décadas.

Atendiendo a las conclusiones derivadas de los estudios expuestos previamente en la tabla anterior, las universidades deben estudiar y adaptar conceptos del marketing como la creación de valor o la formación de una imagen adecuada pues ya no existe un debate en las organizaciones acerca de generar o producir servicios y productos sino que la atención de las organizaciones se dirige en la actualidad en la búsqueda de una calidad superior y una imagen adecuada (Hu et al., 2009) Diversas investigaciones han demostrado que la calidad del servicio, el valor y la imagen son factores claves en la obtención de ventajas competitivas (Bolton y Drew, 1991; Parasuraman, Berry y Zeithaml, 1991; Zeithaml, Berry y Parasuraman, 1996). 


\begin{tabular}{|c|c|c|}
\hline \multicolumn{3}{|c|}{$\begin{array}{c}\text { CUADRO 1: ESTUDIOS SOBRE LA PRÁCTICA DE MARKETING } \\
\text { EN LA EDUCACIÓN SUPERIOR }\end{array}$} \\
\hline Autor y año & $\begin{array}{l}\text { Tipo de } \\
\text { Estudio }\end{array}$ & Aportación \\
\hline Litten (1980) & Predictivo & $\begin{array}{l}\text { La orientación al mercado de las universidades reduce la } \\
\text { dificultad de adaptación ante el nuevo escenario económico y } \\
\text { político. }\end{array}$ \\
\hline Topor (1983) & Exploratorio & $\begin{array}{l}\text { Establece los distintos procesos necesarios para desarrollar una } \\
\text { estrategia de marketing en la educación superior. }\end{array}$ \\
\hline $\begin{array}{l}\text { Conway, } \\
\text { Mackay y Yorke } \\
\text { (1994) }\end{array}$ & Descriptivo & $\begin{array}{l}\text { Como resultado del contexto competitivo, la universidad necesita } \\
\text { de la orientación al marketing a fin de adquirir una ventaja } \\
\text { competitiva sobre sus rivales. Evalúa el grado en que las } \\
\text { instituciones muestran un conocimiento de los distintos tipos de } \\
\text { clientes que necesitan ser tomados en consideración en la } \\
\text { formulación de sus planes de marketing. }\end{array}$ \\
\hline $\begin{array}{l}\text { Taylor y Reed } \\
\text { (1995) }\end{array}$ & Exploratorio & $\begin{array}{l}\text { La orientación al estudiante se conforma como la piedra angular } \\
\text { de una auténtica estrategia de marketing dentro de las } \\
\text { universidades. El concepto de marketing en la ES supone la } \\
\text { redefinición del término dado su peculiar contexto. }\end{array}$ \\
\hline $\begin{array}{l}\text { Williams, Liu y } \\
\text { Shi (1997) }\end{array}$ & Descriptivo & $\begin{array}{l}\text { Estudio del impacto de la orientación al marketing y la } \\
\text { aplicación de tasas a los estudiantes en el contexto chino. } \\
\text { Aumento de la capacidad internacional de las universidades } \\
\text { como consecuencia de las estrategias de marketing. }\end{array}$ \\
\hline $\begin{array}{l}\text { Caruana, } \\
\text { Ramaeshan y } \\
\text { Ewing (1998) }\end{array}$ & $\begin{array}{l}\text { Causal/ } \\
\text { Empírico }\end{array}$ & $\begin{array}{l}\text { Los resultados confirmaron la relación positiva entre la } \\
\text { orientación al mercado y el desempeño universitario mediante la } \\
\text { realización de escala de medida de marketing (MARKOR) en } \\
\text { universidades australianas. }\end{array}$ \\
\hline Mazzarol (1998) & $\begin{array}{l}\text { Causal/ } \\
\text { Empírico }\end{array}$ & $\begin{array}{l}\text { El desarrollo de una ventaja competitiva para la competencia } \\
\text { internacional de la educación a través de estrategias de } \\
\text { marketing es un tema complejo. La educación es un producto } \\
\text { altamente intangible y tiene excesivas particularidades en su } \\
\text { estrategia de marketing. El éxito de una orientación al mercado } \\
\text { internacional es la imagen y las alianzas globales. La estrategia } \\
\text { de marketing aumenta la financiación conseguida por las } \\
\text { universidades. }\end{array}$ \\
\hline $\begin{array}{l}\text { Siu } y \text { Wilson } \\
(1998)\end{array}$ & $\begin{array}{l}\text { Causal/ } \\
\text { Empírico }\end{array}$ & $\begin{array}{l}\text { Apoyo empírico a la puesta en práctica de la orientación al } \\
\text { marketing en tres universidades inglesas. Es fundamental la } \\
\text { coordinación y comunicación con los públicos tanto externos } \\
\text { como internos para lograr una verdadera orientación al mercado. }\end{array}$ \\
\hline $\begin{array}{l}\text { Wasmer } \quad y \\
\text { Bruner (2000) }\end{array}$ & Exploratorio & $\begin{array}{l}\text { Examen de la relación de las características de la universidad } \\
\text { (tamaño, régimen público o privado e innovación) y la } \\
\text { perspectiva de marketing. Mayor orientación en universidades } \\
\text { pequeñas y de tipo privado. }\end{array}$ \\
\hline
\end{tabular}




\begin{tabular}{|c|c|c|}
\hline $\begin{array}{l}\text { Umashankar } \\
\text { (2001) }\end{array}$ & Exploratorio & $\begin{array}{l}\text { Adaptación de las políticas de marketing para la atracción y } \\
\text { retención de estudiantes. Dada la nueva competencia } \\
\text { internacional es necesaria una planificación estratégica de } \\
\text { comunicación e imagen desde la perspectiva del marketing. }\end{array}$ \\
\hline $\begin{array}{ll}\text { Czarniawska } & \text { y } \\
\text { Genell (2002) }\end{array}$ & Exploratorio & $\begin{array}{l}\text { Las estrategias de mercado pueden mejorar la calidad sin } \\
\text { embargo, aunque pueda ser contradictorio, llevará a la pérdida de } \\
\text { identidad de las universidades. }\end{array}$ \\
\hline $\begin{array}{l}\text { Maringe } \quad y \\
\text { Foskett (2002) }\end{array}$ & Descriptivo & $\begin{array}{l}\text { Presentan un estudio sobre la aplicación de teorías de marketing } \\
\text { en las universidades sudafricanas. La orientación al marketing se } \\
\text { basa fundamentalmente en las políticas de ventas (número de } \\
\text { estudiantes) y las relaciones públicas para la gestión de la } \\
\text { imagen. }\end{array}$ \\
\hline $\begin{array}{l}\text { Arnett, German } \\
\text { y Hunt (2003) }\end{array}$ & $\begin{array}{l}\text { Causal/ } \\
\text { Empírico }\end{array}$ & $\begin{array}{l}\text { La orientación al mercado y la filosofía del marketing de } \\
\text { relaciones es una estrategia viable en contextos de alto } \\
\text { intercambio social como es el caso de las universidades. }\end{array}$ \\
\hline Bok (2003) & Descriptivo & $\begin{array}{l}\text { Análisis del impacto de las técnicas de marketing en distintas } \\
\text { áreas de la universidad (servicios deportivos, facultades...). La } \\
\text { orientación al marketing es necesaria para crear el valor de la } \\
\text { universidad, que debe estar basado en la aportación cultural y } \\
\text { debe ser motor del conocimiento de la sociedad. }\end{array}$ \\
\hline Jongbloed (2003) & Exploratorio & $\begin{array}{l}\text { Establece las condiciones para la aplicación del marketing en la } \\
\text { educación superior tanto para los estudiantes como la propia } \\
\text { institución dentro del contexto universitario holandés. }\end{array}$ \\
\hline Trim (2003) & Exploratorio & $\begin{array}{l}\text { Aportación de un marco teórico de un enfoque profesional y } \\
\text { relacional de marketing que garantice que se cumplen las } \\
\text { expectativas del cliente, y que se pueda utilizar para auditar, } \\
\text { evaluar y para la gestión estratégica. }\end{array}$ \\
\hline $\begin{array}{l}\text { Cann y George } \\
(2004)\end{array}$ & Exploratorio & $\begin{array}{l}\text { Establecen un modelo de diagnóstico en base a un modelo entre } \\
\text { la orientación al aprendizaje y la orientación al marketing } \\
\text { pudiendo ser utilizado como una herramienta para evaluar si una } \\
\text { institución logra sus objetivos organizacionales y de gestión. }\end{array}$ \\
\hline $\begin{array}{l}\text { Ivy y Naude } \\
(2004)\end{array}$ & $\begin{array}{l}\text { Causal/ } \\
\text { Empírico }\end{array}$ & $\begin{array}{l}\text { Examen de las políticas de marketing en los MBA en el contexto } \\
\text { sudafricano. El marketing mix de la educación superior debe } \\
\text { estar compuesto por siete P`s: Premium (alta calidad); } \\
\text { Prominencia (imagen adecuada); Publicidad, Precio, Programa } \\
\text { de estudios, Prospectus (fuentes de información) y Personas. }\end{array}$ \\
\hline $\begin{array}{l}\text { Flavián y Lozano } \\
\text { (2004) }\end{array}$ & $\begin{array}{l}\text { Causal/ } \\
\text { Empírico }\end{array}$ & $\begin{array}{l}\text { La orientación al mercado del profesorado universitario de } \\
\text { marketing ejerce un efecto positivo en los resultados obtenidos } \\
\text { en docencia e investigación. }\end{array}$ \\
\hline $\begin{array}{l}\text { Hemsley-Brown } \\
\text { y Oplatka (2006) }\end{array}$ & Exploratorio & $\begin{array}{l}\text { Análisis de los resultados de las políticas de marketing en países } \\
\text { de habla inglesa. La literatura acerca del marketing en las } \\
\text { universidades es incoherente e incompleta y no existen modelos } \\
\text { que expliquen el proceso de orientación al mercado en la } \\
\text { universidad. }\end{array}$ \\
\hline
\end{tabular}




\begin{tabular}{|c|c|c|}
\hline $\begin{array}{l}\text { Marzo, Pedraja } \\
\text { y Rivera (2006) }\end{array}$ & Exploratorio & $\begin{array}{l}\text { Justificación del marketing en el sector público universitario } \\
\text { como consecuencia de su pérdida de monopolio y como vía más } \\
\text { eficaz para satisfacer a los distintos públicos. }\end{array}$ \\
\hline Voon (2006) & $\begin{array}{l}\text { Causal/ } \\
\text { Empírico }\end{array}$ & $\begin{array}{l}\text { Los resultados de su estudio muestran que la orientación al } \\
\text { servicio impulsado por el mercado consta de seis componentes } \\
\text { (orientación al cliente, orientación a los competidores, } \\
\text { orientación interfuncional, orientación hacia los resultados, } \\
\text { orientación a largo plazo, y la orientación de empleados) y que } \\
\text { estos tienen una relación significativamente fuerte y positiva con } \\
\text { la calidad del servicio. }\end{array}$ \\
\hline Hayes (2007) & Descriptivo & $\begin{array}{l}\text { Predicción de las estrategias de marketing de las universidades en } \\
\text { el futuro a través de un panel de } 20 \text { expertos en ES. En el futuro } \\
\text { aumentará la importancia del marketing en la educación } \\
\text { superior. }\end{array}$ \\
\hline Akonkwa (2009) & Predictivo & $\begin{array}{l}\text { Plantea los principales problemas relacionados con el uso de la } \\
\text { orientación al marketing en la educación superior (en términos } \\
\text { de las clásicas 4P’s), y sugiere una agenda de investigación que } \\
\text { mejore la importación de conceptos de marketing en la } \\
\text { educación superior. Urge la coordinación entre financiación, } \\
\text { presupuesto y política de marketing. }\end{array}$ \\
\hline $\begin{array}{l}\text { Maringe y Gibbs } \\
(2009)\end{array}$ & Exploratorio & $\begin{array}{l}\text { Analiza los distintos componentes y técnicas del marketing de } \\
\text { las universidades y establece los distintos métodos para el } \\
\text { correcto posicionamiento de la institución. }\end{array}$ \\
\hline $\begin{array}{l}\text { Newman y Jahdi } \\
\text { (2009) }\end{array}$ & Exploratorio & $\begin{array}{l}\text { El marketing de la educación superior debe considerar el uso de } \\
\text { un marco más amplio proporcionado por la teoría del marketing } \\
\text { de servicios. El marketing de la universidad no es sólo producto, } \\
\text { distribución, precio y publicidad, sino que también son las } \\
\text { personas, los procesos y las pruebas físicas. }\end{array}$ \\
\hline Oplatka (2009) & Descriptivo & $\begin{array}{l}\text { Análisis de las diferencias entre las políticas de marketing entre } \\
\text { las universidades israelíes y las inglesas. En general, los } \\
\text { académicos de ambos países indicaron que su institución se } \\
\text { orienta hacia el marketing ya que se adaptan a las necesidades y } \\
\text { deseos de los estudiantes. Además, los empleados consideran } \\
\text { que contribuyen a la estrategia de marketing pues mejoran la } \\
\text { imagen a través de su servicio prestado al estudiante. }\end{array}$ \\
\hline $\begin{array}{l}\text { Ramachandran } \\
\text { (2010) }\end{array}$ & Exploratorio & $\begin{array}{l}\text { Ofrece una visión crítica del marketing en la ES argumentando } \\
\text { que restringen los derechos y privilegios que los estudiantes y el } \\
\text { sector de la ES tradicionalmente disfrutaban. }\end{array}$ \\
\hline $\begin{array}{l}\text { Hemsley-Brown } \\
\text { y Oplatka (2010) }\end{array}$ & $\begin{array}{l}\text { Causal/ } \\
\text { Empírico }\end{array}$ & $\begin{array}{l}\text { La satisfacción de las necesidades del estudiante, y un enfoque } \\
\text { centrado en el estudiante dentro de la orientación al mercado } \\
\text { debe ser una misión institucional, así como una iniciativa } \\
\text { impulsada por el gobierno hacia las universidades a través de la } \\
\text { introducción de prácticas y principios de marketing. }\end{array}$ \\
\hline
\end{tabular}




\begin{tabular}{|c|c|c|}
\hline $\begin{array}{l}\text { Ross, Grace y } \\
\text { Shao (2013) }\end{array}$ & Descriptivo & $\begin{array}{l}\text { La orientación al marketing aporta beneficios como el aumento } \\
\text { de la satisfacción, la creación de valor para los estudiantes } \\
\text { internacionales, el aumento de la contratación y retención de los } \\
\text { estudiantes además de una mayor cuota de mercado y posición } \\
\text { competitiva en el marco actual. Es consecuencia de la menor } \\
\text { financiación pública gubernamental. }\end{array}$ \\
\hline $\begin{array}{l}\text { Maringe y } \\
\text { Mourad (2012) }\end{array}$ & Exploratorio & $\begin{array}{l}\text { Diferencias entre las políticas de marketing entre los países } \\
\text { desarrollados y los países subdesarrollados. Adaptación del } \\
\text { marketing a la internacionalización de las universidades. }\end{array}$ \\
\hline $\begin{array}{l}\text { Judson y Taylor } \\
\text { (2014) }\end{array}$ & Exploratorio. & $\begin{array}{l}\text { Propuesta de un modelo teórico de aumento de capacidades en el } \\
\text { ámbito de la educación superior y su estrategia de marketing. } \\
\text { Supone para el alumno el aumento de capacidades: sociales, } \\
\text { cognitivas, morales y actitudinales. Todo ello para lograr una } \\
\text { adecuación co-creación del valor en la universidad. }\end{array}$ \\
\hline $\begin{array}{l}\text { Babb } \\
\text { (2015) }\end{array}$ & Exploratorio & $\begin{array}{l}\text { La comercialización de la educación superior está adquiriendo } \\
\text { una atención más formal y que la conciencia del fenómeno se } \\
\text { está ampliando. Sin embargo, la mayor parte del esfuerzo en } \\
\text { marketing se ha centrado en la promoción y venta de programas } \\
\text { para estudiantes potenciales obviando todo el proceso y } \\
\text { experiencia del alumno. }\end{array}$ \\
\hline $\begin{array}{l}\text { Wu y Naidoo } \\
\text { (2016) }\end{array}$ & Exploratorio & $\begin{array}{l}\text { Con la disminución de matrículas y los continuos recortes de } \\
\text { fondos, muchas instituciones de educación superior están } \\
\text { explorando nuevas formas de comercializar y promocionarse a } \\
\text { los estudiantes internacionales. El papel del marketing } \\
\text { internacional en la educación superior es cada vez más } \\
\text { importante. }\end{array}$ \\
\hline
\end{tabular}

En definitiva, en función de lo revisado hasta el momento la universidad debe:

- Integrar las políticas de marketing para lograr relaciones positivas y satisfactorias con todos sus stakeholders. Ello nace como respuesta, entre otros motivos, a las circunstancias y retos actuales de las universidades.

- Desarrollar estrategias que respondan a la sociedad según los principios del marketing de responsabilidad social.

- Alcanzar los objetivos y retos del entorno actual a través de políticas de marketing.

- Conocer las percepciones de sus distintos públicos y lograr una imagen favorable y distintiva.

- Lograr una política de gestión que integre el marketing de relaciones con sus diferentes públicos, un marketing interno dentro de la propia organización y un marketing integrado que coordine las múltiples actividades que debe desarrollar la universidad.

Además, intentando establecer un marco conceptual con base en lo revisado y teniendo en cuenta lo comentado en este trabajo se propone una clasificación de las características que determinan el marketing universitario partiendo de las aportaciones de Kotler y Kartajaya (2010) acerca de la evolución del marketing. 


\begin{tabular}{|c|c|c|c|c|}
\hline \multicolumn{5}{|c|}{ CUADRO 2: COMPETENCIAS DEL MARKETING DE UNIVERSIDADES } \\
\hline COMPETENCIA & $\begin{array}{l}\text { Marketing } 1.0 \\
\text { Centrado en el } \\
\text { producto }\end{array}$ & $\begin{array}{l}\text { Marketing } 2.0 \\
\text { Centrado en el } \\
\text { consumidor }\end{array}$ & $\begin{array}{l}\text { Marketing 3.0 } \\
\text { Orientado a } \\
\text { valores }\end{array}$ & $\begin{array}{l}\text { Marketing } 3.0 \\
\text { Universidades }\end{array}$ \\
\hline Objetivo & Vender productos & 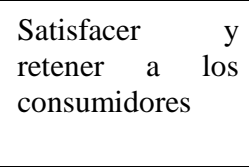 & $\begin{array}{l}\text { Satisfacer y } \\
\text { retener a los } \\
\text { consumidores }\end{array}$ & $\begin{array}{l}\text { Contribuir } \\
\text { conocimiento, al bien } \\
\text { común, al desarrollo } \\
\text { humano y social }\end{array}$ \\
\hline $\begin{array}{l}\text { Factores } \\
\text { favorecedores }\end{array}$ & $\begin{array}{l}\text { Revolución } \\
\text { industrial }\end{array}$ & TIC & $\begin{array}{ll}\text { Nueva } & \text { ola } \\
\text { tecnológica } & \end{array}$ & $\begin{array}{l}\text { Educación más universal } \\
\text { y nuevas tecnologías }\end{array}$ \\
\hline $\begin{array}{l}\text { Cómo se ve a los } \\
\text { ciudadanos }\end{array}$ & $\begin{array}{l}\text { Compradores } \\
\text { masivos con } \\
\text { necesidades físicas }\end{array}$ & $\begin{array}{l}\text { Consumidor } \\
\text { inteligente con } \\
\text { mente y corazón }\end{array}$ & $\begin{array}{l}\text { Ser humano con } \\
\text { mente, espíritu y } \\
\text { corazón }\end{array}$ & $\begin{array}{l}\text { Ser humano con } \\
\text { inquietudes, mente } y \\
\text { espíritu }\end{array}$ \\
\hline Concepto clave & $\begin{array}{ll}\begin{array}{l}\text { Desarrollo de } \\
\text { producto }\end{array} & \\
\end{array}$ & Diferenciación & Valores & $\begin{array}{l}\text { Valores, contenidos y } \\
\text { capacidades }\end{array}$ \\
\hline $\begin{array}{l}\text { Directrices de } \\
\text { marketing de la } \\
\text { campaña }\end{array}$ & $\begin{array}{l}\text { Especificación de } \\
\text { producto }\end{array}$ & $\begin{array}{l}\text { Posicionamiento } \\
\text { del producto y la } \\
\text { compañía }\end{array}$ & $\begin{array}{l}\text { Misión, visión y } \\
\text { valores de la } \\
\text { compañía }\end{array}$ & $\begin{array}{l}\text { Centrada en la formación } \\
\text { de ciudadanos y } \\
\text { profesionales }\end{array}$ \\
\hline $\begin{array}{l}\text { Propuesta de } \\
\text { valor }\end{array}$ & Funcional & $\begin{array}{l}\text { Funcional } \\
\text { emocional }\end{array}$ & $\begin{array}{l}\text { Funcional, } \\
\text { emocional } \\
\text { espiritual }\end{array}$ & $\begin{array}{l}\text { Funcional, emocional, } \\
\text { social y epistémico } \\
\text { (despertar la curiosidad, } \\
\text { proporcionar novedad o } \\
\text { satisfacer un deseo de } \\
\text { conocimiento) }\end{array}$ \\
\hline $\begin{array}{l}\text { Interacción con } \\
\text { los consumidores }\end{array}$ & $\begin{array}{l}\text { Transacción uno- } \\
\text { muchos }\end{array}$ & $\begin{array}{l}\text { Relación uno- } \\
\text { uno }\end{array}$ & $\begin{array}{l}\text { Colaboración } \\
\text { muchos-muchos }\end{array}$ & $\begin{array}{l}\text { Colaboración } \\
\text { muchos-muchos }\end{array}$ \\
\hline
\end{tabular}

\section{CONCLUSIONES}

El objetivo de este trabajo era la comprensión de las estrategias de marketing en la universidad y conocer en profundidad el estado de la cuestión. Para ello se han revisado 104 publicaciones versadas sobre este tema. Entre los resultados alcanzados, en este artículo se ha comprobado que ha habido un crecimiento del interés por desarrollar nuevas estrategias que configuren la relación entre la universidad y sus públicos. Las instituciones universitarias actúan como motor de crecimiento económico pues la educación y la economía se han relacionado tradicionalmente a través del triángulo "educación-crecimiento-desarrollo" (Fernández, Rodeiro y Ruzo, 2005, pág. 77). Así, en esta vertiente, aparece la aplicación del marketing en las universidades. La literatura académica acerca de la adopción de las prácticas de marketing por parte de las universidades es relativamente reciente. Ésta se remonta a principios de los años 80, cuando el marco político y económico obligó a las instituciones a buscar nuevas ideas estratégicas (Maringe y Mourad, 2012).

Estas nuevas prácticas de marketing han surgido como una respuesta a la creciente competitividad y exigencias de los agentes sociales. Hasta la fecha actual los diferentes estudios llevados a cabo sobre el vínculo entre marketing y universidad se han basado, fundamentalmente, en reflexiones o estudios exploratorios acerca del fenómeno. Sin embargo, son escasos los estudios empíricos que demuestren cómo intervienen los componentes del marketing entre sus públicos. Los beneficios que aporta una orientación al marketing se han demostrado mediante resultados de eficacia, de nuevos estudiantes o según la opinión de los gestores. 
Otro "gap" detectado en la literatura es la falta de un marco teórico o teoría conceptual del marketing propia de las universidades, pues los estudios hasta la fecha se han basado en teorías previas de otros sectores de servicios o se han centrado en debatir acerca de si esta óptica es válida, por qué se origina o qué consecuencias tiene la orientación al marketing en el sector público universitario. En este sentido se ha aportado un modelo teórico que sirva para delimitar la orientación al marketing en la universidad. Este modelo comprende los factores internos y externos que afectan a la universidad y que han dado lugar a un nuevo enfoque de gestión con una especial importancia del marketing mix a adoptar por las universidades, integrado por: procesos, personas, capacidades, alta calidad (premium) e imagen (proyección).

Intentando completar ese vacío, desde nuestra perspectiva el marketing de universidades es el análisis de las necesidades e intereses de los distintos públicos y la coordinación del conjunto de acciones dirigidas a mejorar la oferta educativa, la transferencia y el desarrollo científico desde una perspectiva social con el objetivo de crear valor y satisfacer a la sociedad con sus distintos públicos. Se trata de una visión social y relacional del marketing donde se responda a iniciativas culturales o de desarrollo humano a la vez que se oriente a mantener relaciones duraderas con sus clientes.

En torno al concepto de valor, en este trabajo se propone una estrategia de marketing 3.0 adaptada a la idiosincrasia del servicio universitario. El valor que ofrece la universidad a un estudiante pasa por el valor emocional (etapa de importante desarrollo personal), epistémico con la adquisición de conocimientos, valor social, pues las interacciones con sus otros compañeros y la realización de actividades complementarios enriquecen la etapa universitaria, y funcional pues esperan verse recompensados laboralmente el esfuerzo realizado. La adopción de estrategias de marketing por las IES debe estar orientada al desarrollo humano y social aprovechando los factores tecnológicos que promueven una educación más universal. El propósito de la universidad debe girar en torno a una educación que forme a los estudiantes en inquietudes, un ser humano "con mente y espíritu” que se conviertan en capital humano al servicio de la sociedad. Para ello es vital, la colaboración entre todos los agentes implicados, desde las empresas hasta los propios estudiantes. En la educación superior es donde alcanza una mayor importancia la co-creación del servicio (Judson y Taylor, 2014).

Otra problemática detectada en este trabajo es el asunto de los públicos o stakeholders de la universidad ya que es uno de los puntos conflictivos a la hora de delimitar una estrategia de marketing, pues pueden ser muy diversas y diferentes las percepciones de cada uno de ellos. Aunque ha surgido un debate en la literatura acerca del rol del estudiante como cliente o no, nosotros nos situamos en la línea de Mintzberg (1996) que contempla al estudiante como un público fundamental pero que no adquiere las características propias del cliente de cualquier otro campo de consumo. El autor argumenta que en ciertos campos (normalmente públicos) como la educación, servicios medioambientales (por ejemplo, el reciclaje) o la tributación de impuestos, el “cliente” es evaluado según su nivel de responsabilidad o implicación, aspecto que no sucede en otros ámbitos de consumo. La relación de los ciudadanos con las instituciones públicas resulta de gran complejidad y depende del contexto y las aspiraciones de cada persona pudiendo encajar como consumidores, clientes, ciudadanos o sujetos (Mintzberg, 1996). Aunque existe un fuerte debate sobre este aspecto, sií se considera necesario una orientación al "cliente", es decir, a los distintos públicos de la universidad para mejorar el servicio y satisfacer sus necesidades. Por lo tanto, en nuestra opinión, es preferible no denominar al estudiante como cliente, pero sí debe existir una orientación hacia el mismo dentro de la institución. Supone adaptar una filosofía de marketing de satisfacción al cliente sin que sea incompatible con los estándares educativos. Así mismo, 
el estudio de la percepción de unos principales públicos en la actualidad, los egresados (denominados como alumni en el mundo anglosajón) no ha sido suficientemente examinado en la literatura académica. En nuestro estudio, se sugiere denominar a los distintos stakeholders de la universidad como públicos o agentes en lugar de clientes.

En definitiva, el nuevo escenario en que se mueve la universidad ha obligado a orientarse y esforzarse por lograr una ventaja competitiva que les garantice su supervivencia en el mercado de la educación superior (Mora, 1997, Oldfield y Baron, 2000, García-Sanchís, 2012). Podíamos concluir, en función de lo revisado anteriormente, que ha sido la propia evolución de la sociedad la que ha dado lugar a la necesidad de adaptar estrategias de marketing. Por ejemplo, la universidad durante muchos años no tuvo la necesidad de atraer estudiantes dado el incipiente crecimiento de la sociedad, sin embargo, la importancia de la investigación e internacionalización, el aumento del número de universidades y las dificultades de financiación ha obligado a adquirir nuevas tácticas. La implantación de prácticas de marketing resulta fundamental dado el nuevo entorno y los múltiples beneficios que se derivan de ellas. La aportación del marketing en la universidad es fundamentalmente la del análisis de información externa e interna que ayuda en las decisiones estratégicas, así como la sistematización y coordinación de las tareas a realizar para proporcionar un mejor servicio a los distintos públicos.

Progresivamente, las universidades han desarrollado y establecido políticas de marketing en su gestión por lo que ha aumentado progresivamente la temática en esta área. Sin embargo, y como principal futura línea de investigación, se debe contribuir con estudios empíricos que permitan conocer la opinión de los diferentes implicados y, sobre todo, la eficacia de estrategias de marketing en el sector universitario más allá del concepto de calidad.

\section{BIBLIOGRAFÍA}

Akonkwa, D. B. M. (2009): "Is market orientation a relevant strategy for higher education institutions?: Context analysis and research agenda”, International Journal of Quality and Service Sciences, 1 (3), 311-333.

AMA: American Marketing Association (2013): Dictionary, http://www.marketingpower.com/_layouts/Dictionary.aspx?source=footer.

Arena, M., Arnaboldi, M. y Azzone, G. (2010): "Student perceptions and central administrative services: the case of higher education in Italy", Studies in Higher Education, 35 (8), 941-959.

Arnett, D. B., German, S. D. y Hunt, S. D. (2003): "The identity salience model of relationship marketing success: The case of nonprofit marketing”, Journal of Marketing, 67 (2), 89-105.

Arnoldo, H. y Nicolás, M. (2001): Estrategias para el Liderazgo Competitivo, Buenos Aires: Editorial Dolmen.

Babb, H. W., \& Giga, S. (2015): "Marketing in Higher Education: Myth or Reality", In Proceedings of the 1986 Academy of Marketing Science (AMS) Annual Conference (pp. 132-135). Springer, Cham.

Bardo, J. W., Ross, R. H. y Headley, E. L. (1990): "Measuring alumni's image of a university”, Journal for Higher Education Management, 6 (1), 29-44. 
Beerli Palacio, A., Díaz Meneses, G. y Pérez Pérez, P. J. (2002): “The configuration of the university image and its relationship with the satisfaction of students”, Journal of Educational Administration, 40 (5), 485-505.

Blackburn, J. C. (1980): “Marketing in Admissions: A Perspective on Its Use”, College Board Review, 116, 18-21.

Bok, D. (2003): Universities in the marketplace, New Jersey: Princeton University Press.

Bolton, R. N. y Drew, J. H. (1991): “A multistage model of customers' assessments of service quality and value”, Journal of consumer research, 17 (4), 375-384.

Bolton, R. N. y Lemon, K. N. (1999): “A Dynamic Model of Customers' Usage of Services: Usage as an Antecedent and Consequence of Satisfaction”, Journal of Marketing Research, 36 (2), 171-186.

Booms, B. H. y Bitner, M. J. (1981): "Marketing strategies and organisation structures for service firms", en Marketing of Services, Ed. Donnelly, J. H and George, W. R. Chicago: American Marketing Association, pp. 47-51.

Cann, C. W. y George, M. A. (2004): "Key elements of a successful drive toward marketing strategy making”, Journal of Marketing for Higher Education, 13 (1-2), 1-15.

Capelleras, J. l. y Veciana, J. M. (2004): “Calidad de servicio en la enseñanza universitaria: desarrollo y validación de una escala de medida”, Revista Europea de Dirección y Economía de la Empresa, 13 (4), 55-72.

Caruana, A., Ramaseshan, B. y Ewing, M. T. (1998): "The market orientation-performance link: some evidence from the public sector and universities”, Journal of Nonprofit \& Public Sector Marketing, 6 (1), 63-82.

Clark, B. (1998): Creating entrepreneurial universities: organizational pathways of transformation, England: Elsevier Science Ltd.

Conway, T., Mackay, S. y Yorke, D. (1994): "Strategic planning in higher education: Who are the customers”, International Journal of Educational Management, 8 (6), 29-36.

Czarniawska, B. y Genell, K. (2002): "Gone shopping? Universities on their way to the market”, Scandinavian Journal of Management, 18 (4), 455-474.

Dale, R. (2007): "Specifying globalization effects on national policy”, The Routledge Falmer Reader in Education Policy and Politics, 14 (1), 1-17.

David, F. R. (2003): Conceptos de administración estratégica, Pearson Educación.

DeShields Jr, O. W., Kara, A. y Kaynak, E. (2005): "Determinants of business student satisfaction and retention in higher education: applying Herzberg's two-factor theory”, International journal of educational management, 19 (2), 128-139.

Díaz Barriga, A. (1998): Organismos internacionales y política educativa, Ciudad de México: Siglo XXI Editores

Díaz-Canel, M. (2011): Discurso en la UNESCO, Paris. 1 Octubre.

Dolinsky, A. L. (1994): “A consumer complaint framework with resulting strategies: An application to higher education”, Journal of Services Marketing, 8 (3), 27-39.

Eagle, L. y Brennan, R. (2007). “Are students customers? TQM and marketing perspectives”, Quality Assurance in Education, 15 (1), 44-60.

Fernández López, S., Rodeiro Pazos, D. y Ruzo Sanmartín, E. (2005): “La lucha por la supervivencia: competitividad de la oferta de las universidades”, Revista Europea de Dirección y Economía de la Empresa, 14 (1), 77-94. 
Flavián, C. y Lozano, F.J. (2004): La influencia del ambiente en la relación entre orientación al mercado y los resultados en el contexto universitario español, en XIV Congreso ACEDE (Asociación Científica de Economía y Dirección de la Empresa).

García Sanchís, M. (2012): "Evaluación del servicio universitario. Una aproximación integradora a partir de la calidad, el valor y la satisfacción, desde un enfoque crosscultural". Tesis Doctoral, Universitat de València.

Gibbs, P., \& Knapp, M. (2002): Marketing higher and further education: An educator's guide to promoting courses, departments and institutions, Psychology Press.

Hayes, T. (2007): "Delphi study of the future of marketing of Higher Education”, Journal of Business Research, 60 (9), 927-931.

Hemsley-Brown, J. y Oplatka, I. (2006): "Universities in a competitive global marketplace: A systematic review of the literature on higher education marketing”, International Journal of Public Sector Management, 19 (4), 316-338.

Hemsley-Brown, J. y Oplatka, I. (2010): "Market orientation in universities: A comparative study of two national higher education systems”, International Journal of Educational Management, 24 (3), 204-220.

Helgesen, Ø. y Nesset, E. (2007): “Images, satisfaction and antecedents: Drivers of student loyalty? A case study of a Norwegian university college”, Corporate Reputation Review, 10 (1), 38-59.

Homburg, C. y Pflesser, C. (2000): “A multiple-layer model of market-oriented organizational culture: measurement issues and performance outcomes”, Journal of Marketing Research, 37 (4), 449-462.

Hu, H. H., Kandampully, J. y Juwaheer, T. D. (2009), "Relationships and impacts of service quality, perceived value, customer satisfaction, and image: an empirical study”, The Service Industries Journal, 29 (2), 111-125.

Hult, G. T. M. y Ketchen, D. J. (2001): "Does market orientation matter?: A test of the relationship between positional advantage and performance”, Strategic management journal, 22 (9), 899-906

Hutton, J. G. (2002): “Narrowing the concept of marketing”, Journal of Nonprofit \& Public Sector Marketing, 9 (4), 5-24.

Ivy, J. y Naude, P. (2004): "Succeeding in the MBA marketplace: identifying the underlying factors”, Journal of Higher Education Policy and Management, 26 (3), 401-417.

Jaworski, B. J. y Kohli, A. K. (1996): "Market orientation: review, refinement, and roadmap”, Journal of Market-Focused Management, 1 (2), 119-135.

Jongbloed, B. (2003): "Marketisation in Higher Education, Clark's triangle and the essential ingredients of markets”, Higher Education Quarterly, 57 (2), 110-135.

Joseph, M. y Joseph, B. (1997): “Service quality in education: a student perspective”, Quality Assurance in Education, 5 (1), 15-21.

Judson, K. M., \& Taylor, S. A. (2014): "Moving from marketization to marketing of higher education: The co-creation of value in higher education", Higher Education Studies, 4 (1), 51-67.

Khalifa, A. (2010): "Rethinking the current dominant approach to business school strategy”, European Business Review, 22 (6), 591-607. 
Kirca, A. H., Jayachandran, S. y Bearden, W. O. (2005): "Market orientation: a meta-analytic review and assessment of its antecedents and impact on performance", Journal of Marketing, 69 (2), 24-41.

Kirp, D. L. (2003): Shakespeare, Einstein and the bottom line: the marketing of Higher Education, USA: Harvard University Press

Kohli, A. K. y Jaworski, B. J. (1990): "Market orientation: the construct, research propositions, and managerial implications”, The Journal of Marketing, 54 (2), 1-18.

Kotler, P. (1972): “A generic concept of marketing”, Journal of Marketing, 36 (2), 46.54.

Kotler, P. (2000): Marketing management, New Jersey: Prentice Hall.

Kotler, P. y Fox, K. (1985): Strategic Marketing for Educational Institutions. Englewood Cliffs, NJ: Prentice Hall.

Kotler, P. y Kartajaya, H. (2010): Marketing 3.0, Ciudad de México: LID Editorial.

Lafferty, B. A. y Hult, G. T. M. (2001): “A synthesis of contemporary market orientation perspectives”, European Journal of Marketing, 35 (1/2), 92-109.

Litten, L. H. (1980): "Marketing Higher Education: Benefits and risks for the American Academic System”, The Journal of Higher Education, 51 (1), 40-59.

Luque Martínez, T. y Del Barrio García, S. (2005): Plan Estratégico Universidad de Granada, Granada: Editorial Universidad de Granada.

Luque-Martínez, T. y Del Barrio-García, S. (2009): "Modelling university image: The teaching staff viewpoint”, Public Relations Review, 35 (3), 325-327.

McCarthy, E.J. y. Perreault, W. D. (2007): Marketing: Planeación Estratégica de la Teoría a la Práctica, 1er. Tomo. McGraw Hill.

McPherson, M. y Schapiro, M. (1998): The Student Aid Game, Princeton, NJ. Princeton University Press.

Margison, S. y Considine, M. (2000): The enterprise university: Power, governance and the reinvention in Australia, Cambridge: Cambridge University press.

Marginson, S. (1997): Markets in Education, St Leonards (Nueva Gales del Sur): Allen \& Unwin.

Maringe, F. (2004): "Vice chancellors' perceptions of university marketing: a view from universities in a developing country”, Higher Education Review, 36 (2), 53-68.

Maringe, F. (2005): "Interrogating the crisis in higher education marketing: the CORD model”, International Journal of Educational Management, 19 (7), 564-578.

Maringe, F. y Foskett, N. H. (2002): "Marketing university education: the South African experience”, Higher Education Review, 34 (3), 35-51.

Maringe, F. y Gibbs, P. (2009): Marketing Higher Education. Theory and Practice, New York: Editorial McGraw- Hill.

Maringe, F. y Mourad, M. (2012): "Marketing for Higher Education in Developing Countries: emphases and omissions”, Journal of Marketing for Higher Education, 22 (1), 1-9.

Marzo, M., Pedraja, M. y Rivera, P. (2006): “¿Se puede aplicar el marketing a los servicios públicos? Justificación en el caso de la Educación Superior”, Revista Aragonesa de Administración Pública 28, 351-369.

Mazzarol, T. (1998): "Critical success factors for international education marketing”, International Journal of Educational Management, 12 (4), 163-175. 
McGrath, J.J. (2003): Leading in a managerialist paradigm: A survey of perceptions within a faculty of education, EdD thesis, Univ. of Birmingham. DOI: http://www.education.bham.ac.uk/programmes/research/documents/McGrath_Thesis.p df.

Michavila, F. (2001): “¿Soplan vientos de cambios universitarios?”, Boletín de la RED-U (Red Estatal de Docencia Universitaria), 1 (1).

Mintzberg, H. (1996): “Managing government, governing management”, Harvard Business Review, 74 (3), 75-83.

Mora, J. G. (1997): "Market trends in Spanish higher education”, Higher Education Policy, 10 (3-4), 3-4.

Munuera, J. L. y Rodríguez Escudero, A. I. (2007): Estrategias de marketing. Un enfoque basado en el proceso de dirección, Madrid: ESIC.

Narver, J. C. y Slater, S. F. (1990): "The effect of a market orientation on business profitability”, Journal of Marketing, 54 (4), 20-35.

Naude, P., y Ivy, J. (1999): "The marketing strategies of universities in the United Kingdom”, International Journal of Educational Management, 13 (3), 126-136.

Newman, C. M. (2002): “The current state of marketing activity among higher education institutions", Journal of Marketing for Higher Education, 12 (1), 15-29.

Newman, S. y Jahdi, K. (2009): "Marketisation of education: Marketing, rhetoric and reality", Journal of Further and Higher Education, 33 (1), 1-11.

OCDE (2013): Education at a Glance 2013: OECD Indicators, OECD, DOI: http://dx.doi.org/10.1787/eag-2013-en

OCDE (zs): Education at a Glance 2016: OECD Indicators, OECD, DOI: http://www.oecd.org/edu/education-at-a-glance-19991487.htm

Oldfield, B. M. y Baron, S. (2000): "Student perceptions of service quality in a UK university business and management faculty”, Quality Assurance in Education, 8 (2), 85-95.

Oplatka, I. (2009): "Marketing the university: The subjective perceptions of Israeli academics of their role in attracting new students to their institution", Journal of Higher Education Policy and Management, 31 (3), 207-217.

Ordorika, I. (2008): “Compromiso con la sociedad: desafíos contemporáneos para las universidades públicas de investigación”, en La educación superior en el mundo, 3, Red Global de Universidad para la Innovación (GUNI), New York: Palgrave Macmillan.

Owlia, M. S. y Aspinwall, E. M. (1997): “TQM in higher education - a review”, International Journal of Quality and Reliability Management 14 (5), 527-543.

Parasuraman, A., Berry, L. L. y Zeithaml, V. A. (1991): "Refinement and reassessment of the SERVQUAL scale”, Journal of Retailing, 67 (4), 420-450.

Parameswaran, R. y Glowacka, A. E. (1995): "University image: an information processing perspective", Journal of Marketing for Higher Education, 6 (2), 41-56.

Pereira, M. A. C. y Da Silva, M. T. (2003): “A key question for higher education: Who are the customers", en Proceedings of the 31st Annual Conference of the Production and Operations , pp. 1-16.

Perin, M. G., Sampaio, C. H., Simões, C. y de Pólvora, R. P. (2012): “Modeling antecedents of student loyalty in higher education”, Journal of Marketing for Higher Education, 22 (1), 101-116. 
Pulido, A. (2009): El futuro de la Universidad. Un tema para debate dentro y fuera de las universidades, Madrid, Delta Publicaciones.

Qureshi, S. (1993): “Market driven public institutions attract resources”, Journal of Professional Services Marketing, 9 (2), 83-92.

Ramachandran, N. T. (2010): "Marketing framework in higher education: Addressing aspirations of students beyond conventional tenets of selling products”, International Journal of Educational Management, 24 (6), 544-556.

Readings, B. (1996): The University in Ruins, Cambridge (Massachusetts); Harvard University.

Redding, P. (2005): “The evolving interpretations of customers in higher education: empowering the elusive”, International Journal of Consumer Studies, 29 (5), 409-417.

Ressler, J. y Abratt, R. (2009): “Assessing the impact of university reputation on stakeholder intentions”, Journal of General Management, 35 (1), 35-45.

Ross, M., Grace, D. y Shao, W. (2013): “Come on higher ed... get with the programme! A study of market orientation in international student recruitment”, Educational Review, 65 (2), 219-240.

Santesmases Mestre, M. (1996): Términos de marketing. Diccionario-Base de Datos, Madrid: Ediciones Pirámide.

Sirvanci, M. (1996): “Are students the true customers of higher education?”, Quality Progress, 29 (10), 99-102.

Sirvanci, M. B. (2004): “Critical issues for TQM implementation in higher education”, The TQM Magazine, 16 (6), 382-386.

Siu, N. Y., y Wilson, R. M. (1998): "Modelling market orientation: An application in the education sector”, Journal of Marketing Management, 14 (4), 293-323.

Slaughter, S. y Leslie, L.L. (1997): Academic Capitalism: Politics, Policies, and the Enterpreneurial University, Baltimore (Maryland): Johns Hopkins University.

Smith, P. (1997): “Marketing adult education: An ideological dilemma?" DOI: http://www.leeds.ac.uk/educol/documents/0000278998.htm

Steger, M. (2003): Globalisation, a very short introduction, Oxford: Oxford university press.

Steiner, G. A. (1979): “Contingency theories of strategy and strategic management”, Strategic management, 405-16.

Sursock, A., Smidt, H. y Davies, H. (2010): Trends 2010: A decade of change in European Higher Education, Brussels: European University Association.

Svensson, G. y Wood, G. (2007): “Are university students really customers? When illusion may lead to delusion for all!”, International Journal of Educational Management, 21 (1), 17-28.

Taylor, R. E. y Reed, R. R. (1995): “Situational Marketing: application for Higher Education Institutions”, Journal of Marketing for Higher Education, 6 (1), 23-36.

Topor, R. (1983): Marketing higher education: a practical guide, (Online), DOI: http://www.marketinged.com/library/index.html\#books

Trim, P. R. (2003): "Strategic marketing of further and higher educational institutions: partnership arrangements and centers of entrepreneurship”, International Journal of Educational Management, 17 (2), 59-70. 
Umashankar, V. (2001): “The Importance of Managing Points-Of-Marketing in Marketing Higher Education Programmes-Some Conclusions”, Journal of Services Research, 1 (1), 125-140.

UNESCO (1998): Declaración en la Conferencia Mundial sobre Educación Superior, París.

Voon, B. H. (2006): "Linking a service-driven market orientation to service quality", Managing Service Quality, 16 (6), 595-619.

Wasmer, D. J. y Bruner, G. C. (2000): "The antecedents of the market orientation in higher education”, Journal of Marketing for Higher Education, 9 (2), 93-105.

Williams, G., Liu, S. S. y Shi, Q. (1997): "Marketization of higher education in the People's Republic of China”, Higher Education Policy, 10 (2), 151-157.

Wonders, T. J. y Gyure, J. F. (1991): “Opportunistic marketing in higher education”, Journal of Marketing for Higher Education, 3 (2), 1-16.

Wu, T. y Naidoo, V. (2016): “The Role of International Marketing in Higher Education”, en International Marketing of Higher Education (pp. 3-9), Palgrave Macmillan US.

Zammuto, R. F., Keaveney, S. M. y O'Connor, E. J. (1996): "Rethinking student services: assessing and improving service quality", Journal of Marketing for Higher Education, 7 (1), 45-70.

Zeithaml, V. A., Berry, L. y Parasuraman, A. (1996): "The Behavioral Consequences of Service Quality”, Journal of Marketing, 60 (2), 31-46. 